\title{
Biomechanics of the osseous pelvis and its implication for consolidative treatments in interventional oncology
}

\begin{abstract}
The osseous pelvis is a frequent site of metastases. Alteration of bone integrity may lead to pain but also to functional disability and pathological fractures. Percutaneous image-guided minimally-invasive procedures, such as cementoplasty and screw fixation, have emerged as a viable option to provide bone reinforcement and fracture fixation, as stand-alone or combined techniques. Understanding the biomechanics of the osseous pelvis is paramount to tailor the treatment to the clinical situation. The purpose of the present review is to present the biomechanics of the osseous pelvis and discuss its implication for the choice of the optimal consolidative treatment.
\end{abstract}

\section{Introduction}

The pelvis is the second most frequent site of osseous metastases after the spine [1]. Alteration of bone integrity in the pelvic girdle may not only lead to pain but also to functional disability and pathological fractures, which in turn can greatly impair the patient's quality of life [2]. Moreover, confinement to bed rest seriously exposes the patient to medical complications, such as thromboembolic and infectious adverse events. External beam radiation and stereotactic body radiation therapy are effective tools to alleviate the pain, however, they do not address the mechanical instability [3,4]. A wide range of open surgical procedures have been developed to manage fractures and impending fractures of the pelvic girdle, mostly involving the acetabulum and the proximal femur $[5,6]$. However, the ability to perform these 
potentially complex procedures depends on the general condition of the patient, as the morbidity is not negligible [7]. Image-guided percutaneous minimally-invasive procedures have emerged as a viable option for the treatment of pelvic bone metastases [8]. Among the different tools, percutaneous cementoplasty and screw fixation can provide bone reinforcement and fracture fixation, as stand-alone or combined techniques [2,8-10]. In addition to the patient's condition and clinical objective, the choice of approach and technique should be tailored to the location and features of the lesion. Clinical, anatomical and biomechanical considerations are of utmost importance when considering the different consolidative treatment options. The primary objective of this manuscript is to present a comprehensive review of the biomechanics of the osseous pelvis and its therapeutic implications for the interventional radiologist.

\section{Osseous pelvis - generalities}

The bony pelvis is formed by the pelvic (or coxal, hip or innomate) bones on each side and the sacrum in the midline [11]. The pelvic bones are attached to each other at the level of the pubic symphysis anteriorly and are firmly joined to the sacrum via the sacro-iliac joints and ligaments posteriorly [11]. Some authors also include the proximal femur as part of the bony pelvis $[2,12,13]$. The proximal femur will not be included for the sake of this present review.

\section{Biomechanics}

The pelvic bone is mainly composed of low-density trabecular bone surrounded by a thin layer of high strength cortical bone which mimics the sandwich construction used in composite material, in which the majority of the weightbearing load is carried by a thin shell of 
a high-modulus material with a low-weight core material acting as a spacer $[12,14]$. In the pelvic girdle, the stresses are predominantly transferred through the cortical bone, with a ratio of approximatively 50:1 compared to the underlying trabecular bone [12]. The ligaments and cartilage of the sacro-iliac and hip joints increase the stability of the bony pelvis and the surrounding pelvic musculature help to maintain the stresses within the pelvic bone fairly constant during walking $[12,15,16]$.

The pelvic bone is an almost immobile weight-bearing structure that transfers the gravitational and external loads from the spine to the lower limbs across the sacro-iliac and hip joints (fig.1) [12]. Load transfer across the bony pelvis varies during gait (fig.2). The periacetabular area is the most important weight-bearing structure of the osseous pelvis while the sacro-iliac junction, the pubic region, the iliac wing and the ischium have less weightbearing function [1]. These principles apply to the need of surgical reconstruction following partial pelvic resection. Most of the surgical series do not recommend reconstructions following resection of pelvic bone lesions except if the acetabulum is involved $[1,7]$. Reconstruction of the sacro-iliac joint is more controversial without a consensus [18]. Lesions involving both the sacro-iliac joint and the acetabulum completely interrupt the transmission of stresses and require extensive and often complex reconstruction [19].

Stresses applied to the pelvis vary greatly depending on the location and include compression, tensile, bending and shear forces. Compression is the prevailing stress in the upper part of S1, the anterior part of the sacro-iliac joint and the acetabular roof $[15,20,21]$.

\section{Implications for percutaneous treatment}

Understanding the biomechanical load and distributions in the bony pelvis is critical. Contrary to the long bones of the limbs, there is currently no validated score to predict the risk 
of fracture within the bony pelvis [1,3]. Indications and type of consolidation have to be tailored to each patient and result from several items such as clinical presentation (no symptoms vs mechanical pain), localisation/size/type of lesion, associated destruction of bony cortex, previous local treatments that could weaken the bone resistance (e.g. radiation therapy and thermal ablation), and presence of a pathological fracture (fig.3). Large osseous defects not only increase risk of local mechanical failure but also increase the risk of distant fractures due to the redistribution of the loads within the pelvis [22].

Polymethylmethacrylate (PMMA) bone cement has excellent resistance to compressive forces and is indicated in all areas which are mostly submitted to compression stresses [23,24]. Whenever possible, cement should fill as much as possible the destructed area to restore the transmission of loads and avoid secondary fracture $[10,25,26]$. On the other hand, cement has limited resistance to other stresses than compression and act more as a grout than as alue, which limits its use for the fixation of pelvic fractures [2,24]. To overcome these limitations, percutaneous screw fixation has been described more recently for the management of pathological fractures of the pelvis and the prophylactic stabilization of impending fracture of the proximal femur [2,9,27-29]. Screws have also been used to provide additional support for cement packing and subsequently improve the transmission of loads through the bony pelvis with the analogy of the framework of a concrete construction $[2,29,30]$.

\section{Sacral promontory}

\section{Biomechanics}

The sacral promontory is key for load transmission as it connects the lumbar spine to the rest of the sacrum and subsequently the pelvic bone. Similar to other spinal levels, most of 
the load is sustained by the vertebral body [31]. Compared to the other sacral levels (S2 to S5), the body of S1 is permanently heavily stressed mostly with compression loads [16].

\section{Implications for percutaneous treatment}

Any bone destruction involving the sacral promontory is at risk of mechanical disability and fracture, even though there is no clear critical amount defined in the literature. Consolidation has to be considered in cases of extensive osteolysis, especially if the cortical bone is involved. Cementoplasty is feasible through a transpedicular or a trans-iliac approach and may allow to provide consolidation like for other spinal levels [32,33].

\section{Sacro-iliac joints and sacral ala}

\section{Biomechanics}

The design of the sacro-iliac joint can be assimilated to the one of a flat joint $[21,34]$. Flat surfaces have the optimal design to transfer great moments of mechanical forces, especially compression which is the prevailing stress at the anterior and middle part of the sacro-iliac joint (fig.4A\&4B) [15,34]. Due to their anatomical design, the sacro-iliac joints act as a major stress transmitter and reliever within the bony pelvis. Tensile stresses are maximal in the dorsal and cranial part of the sacro-iliac joint (fig.4C\&4D) [15,34]. The stability of each sacro-iliac joint is therefore enhanced by a process described as "force closure": a perpendicular compressional reaction force to the sacro-iliac joint is generated through (1) a self-bracing mechanism that comes with the conformity and high friction coefficient of adjacent iliac and sacral articular surfaces and (2) the strong binding posterior sacro-iliac ligaments and muscles (piriformis, 
gluteus maximus) $[21,34,35]$. On the other hand, flat joints are particularly vulnerable to dislocation by shear stresses near the joint [34]. However, the sacro-iliac joint can counteract shear stresses, provided that they are firmly pressed together via force closure $[34,35]$. Each sacro-iliac joint has limited mobility, known as nutation. The sacro-iliac joint exhibit a greater range of motion in women than men, potentially explaining the higher rate of fractures of the sacral ala in the female population [34].

\section{Implications for percutaneous treatment}

The sacro-iliac junctions are mechanically important as they account for the transfer of great moments of compression from the trunk to the acetabular area in load-bearing conditions (sitting, standing). If resection without reconstruction is feasible, lumbopelvic instability can occur, and many advocate the need for post-operative reconstruction [18]. In cancer patients with advanced disease, large open surgical procedures are typically not an option due to the morbidity and their invasiveness. Minimally invasive percutaneous interventions are therefore more and more offered as an alternative solution.

Fractures/osteolysis occurring in the anterior part of the sacral ala and associated with functional impairment can be treated with percutaneous cementoplasty (sacroplasty), as compression is the dominant stress in this location (fig.5A,5B,5C). Sacroplasty is superior to non-surgical management to restore patient mobility in case of sacral insufficiency fracture, a frequent condition in cancer patients [36,37]. A recent meta-analysis involving more than 800 patients reported the effectiveness of sacroplasty to manage osteoporotic and malignant sacral fractures, with sustained benefits at 12 months [38]. Alternatively to cement injection, screw fixation of insufficiency/pathological fractures of the sacral ala has demonstrated good functional results requiring a trans-iliac approach in order to approach the fracture line 
perpendicularly $[2,39]$. Based on cadaveric and finite element models, the strongest fixation technique seems to be obtained with the insertion of two parallel screws (i.e that each screw goes through the right and left sacro-iliac joints), one in S1 and one in S2 [40,41]. Because of the underlying bone fragility, the authors usually recommend augmenting the anchorage of the tip of the screws with cement, in order to prevent screw loosening and dislodgement $[29,30]$. There has been no study to date comparing screw fixation and sacroplasty for the management of nondisplaced fractures of the sacral ala. Osteolysis/fracture of the posterior and upper part of the sacro-iliac joint decreases the resistance to tension and shear leading to failure of the strong posterior ligamentous support increasing the risk of pelvic instability. In this situation, cementoplasty might not be sufficient due to poor resistance to shear forces and trans-sacroiliac screw fixation seems mandatory to prevent biomechanical failure, provided that the screws can be anchored in normal trabecular/cortical bone (fig.5D,5E,5F) [24]. Cement augmentation of the screws in the setting of advanced osteolysis may reduce failure of the fixation. $[9,29,30]$. There is limited evidence to support prophylactic stabilization of asymptomatic sacro-iliac lesions, especially if there is no cortical disruption or involvement of the posterior and superior part of the sacro-iliac joint [1].

\section{Acetabulum}

\section{Anatomy and Biomechanics}

The acetabulum is the most important structure of the pelvic bone for load transmission. The supra-acetabular area (i.e the superior and lateral part of the of the acetabulum, also called the acetabular roof) transmits most of the loads and therefore represents the main mechanical bearing part of the acetabulum, regardless the loading conditions (sitting, standing, walking) 
$[12,16,42]$. In particular, the transfer of the hip joint force to the sacro-iliac joint and to the pubic symphysis occurs predominantly through the lateral cortex located above the acetabulum (fig.6) $[16,43]$. Consolidation should always be considered in case of an osteolysis in that region, even if the lesion is asymptomatic. The highest stresses distribution among the trabecular bone is located at the central part of the acetabulum, where the applied forces remain low compared to the supra-acetabular area [12]. The stress concentration at the anterior part of the acetabulum mostly happens during the weight shift and swing phases of gait $[12,16]$. The posterior acetabulum is the weakest area in terms of load transmission $[12,16,42]$. The acetabular cartilage contributes to the distribution of the loads more evenly over the acetabulum [43]. The action of muscle and hip joint forces produce compressive and tensile stresses within

the acetabulum. Overall, compressive stresses predominate on the lateral side and can be considered as high, while tensile stresses occur more on the medial side, the infero-posterior the supero-anterior quadrants of the acetabulum, and appear to be much lower [43].

\section{Implications for percutaneous treatment}

Cancer lesions located in the acetabulum have to be considered at high-risk of mechanical failure, as they may compromise the transmission of forces and lead to disastrous functional complications. Many different surgical options have been developed over the years to reconstruct the acetabulum related to primary/metastatic lesion(s) with or without pathologic fractures $[1,7,44]$. Open surgery usually combines curettage of the tumor prior to consolidation, followed by post-operative radiation. Lesions involving exclusively the acetabular roof can be surgically treated with cemented total hip arthroplasty [2]. Lesions involving the medial wall will require the additional use of a reinforcement ring to avoid medial migration and lesions presenting with extensive cortical destruction are usually treated with total hip replacement 
reinforced by cement and acetabular reconstruction placing pins/screws from the acetabulum into an intact ilium to increase the strength of the anchorage [1,45]. Even though the functional outcomes of open surgery are excellent, they can be very morbid and associated with a high rate of complications (especially for complex reconstructions) and is therefore often not feasible in frail patients or in those patients in which systemic therapy cannot be discontinued $[1,7]$. Thus, percutaneous procedures provide a viable therapeutic option provided that they are adapted to the situation.

Percutaneous cementoplasty can provide efficient consolidation for lesions exclusively involving the acetabular roof ideally without disruption of the articular cortex (fig.7A\&7B) $[6,23]$. This would be analogous to the use of cement in cemented arthroplasty. A finite element analysis demonstrated that for this location complete filling of an acetabular defect, including those with involvement of the lateral cortices, with cement has the potential to restore the transmission of loads to a normal level [26]. The osteolysis should therefore always be filled with as much cement as possible to avoid secondary collapse [10,25]. Ablation prior to cement injection is technically feasible in that location and might also have a role for local tumor control in addition bone consolidation and stabilization [10,46]. It has been reported that complete tumor ablation was a predictive factor of pathologic acetabular fracture stabilization, most likely because it impedes the progression of the osseous destruction [46]. Another potential benefit of ablation is the creation of a cavity-like area that favour lesion filling while potentially reducing the risk of cement leakage. On the other hand, care should be taken not to overablate the surrounding normal bone/cartilage, as this might lead to post-ablation fracture and hip chondrolysis/osteonecrosis [47]. Some authors advocate the additional use of balloon kyphoplasty following ablation and prior to cement injection in order to increase the chance of optimal acetabular roof filling and to prevent intra-articular leakage of cement (fig.7C-7G) [10]. This could be considered analogous to tumor curettage. The reinforcement of cement with 
pins/screws for lesions located in the acetabular roof is increasing being reported, even though there is no study demonstrating the superiority of additional hardware reinforcement versus cement alone for that specific indication [29]. Keeping in mind that the contact between the hardware and cement should be as large as possible in order to effectively transmit the loads [48]. In the case of a non-displaced acetabular pathological fracture, the use of screw fixation allows fixation of the fragments and theoretically improves the overall functional result over stand-alone cementoplasty $[2,9]$.

For extensive osteolysis with or without fracture involving more than just the acetabular roof, percutaneous consolidative techniques will have limited results for consolidation (fig.8). In particular, lesions that destroy both the cortical bone of the roof and the medial wall of the acetabulum expose the patient to a very high risk of mechanical failure and protrusion of the femur inside the pelvis $[1,44,45]$. Surgery should therefore always be considered in those cases. If the patient is not a surgical candidate, percutaneous cementoplasty $+/-$ screw fixation and thermal ablation might be used for palliation with reserved expectations for stabilization outcomes (fig.9) [2,49].

\section{The pubic area}

\section{Biomechanics}

The ilio- and ischio-pubic rami and the pubic symphysis have limited weight-bearing function and participate to load transmission essentially during the weight shift and swing phases of walking [16]. At the pubic symphysis, tensile stress is found at the superior and anterior aspect of the pubic arch, while compressive stress occurs at the inferior and posterior 
aspect of the arch [15]. This results in bending moments in the intermediate region of the pubic symphysis [15]. Because of their orientation and their support for multiple muscular insertions, the ischio- and ilio- pubic rami are submitted to complex stresses including bending and shear [11].

\section{Implications for percutaneous treatment}

The pubic rami have little participation in the transmission of mechanical loads within the pelvis and prophylactic stabilization in an asymptomatic patient suffering from a pubic bone osteolytic lesion or fracture is not mechanically necessary. In the traumatic and oncologic surgical literature, fixation/reconstruction of a unilateral pubic fracture/bone lesions are typically not performed $[1,50]$.

In the case of a mechanically symptomatic osteolysis, interventional radiology offers an effective minimally invasive solution [2,9]. Similarly to trauma, percutaneous screw fixation is an effective technique to achieve prompt stabilization, and is usually performed with a retrograde transpubic ascending access (fig.9) [2,9,51]. Cement may be added to reinforce the anchorage of the hardware, but cementoplasty alone will not be as effective as screw fixation in terms of mechanical reinforcement due to its weak resistance to shear forces $[2,9,24,29]$.

\section{The iliac wing}

\section{Biomechanics}


The iliac wing has very little bearing function and does not participate to the transmission of loads $[1,16]$. However, it still sustains complex stresses during walking because of the insertions of various groups of muscles with strong functional activities [11].

\section{Implications for percutaneous treatment}

Osteolysis located in the iliac wing does not compromise the transmission of loads throughout the pelvis and prophylactic consolidation is usually not necessary [1]. However, extensive cortical and cancellous bone loss in this area may increase the risk of fracture of the iliac wing similar to that seen following bone harvesting for orthopedic \& spine surgeries [52]. In particular, bone loss at the level of the anterior superior iliac spine seems to increase the risk of fracture [52,53]. Cementoplasty is not effective to fix a pathological fracture of the iliac wing because of the complex involved stresses and the inability of cement to fix two bony fragments together [2,24]. Percutaneous screw fixation is an excellent tool to consolidate a pathological/post-ablation iliac wing fracture, allowing early mobilization of the patient (fig.10) $[2,9,54,55]$. Care should be taken to use long screws bridging the fracture line as perpendicular as possible with both extremities anchored in normal bone. The addition to cement might allow to increase the stability of the screw should the landing zone of one extremity of the screw be located in bone of poor underlying quality (e.g. osteopenia, post-radiation/ablation) [2,9,29].

\section{Conclusion}

The sacral promontory and the acetabular roof are the most critical mechanical portions of the pelvis, mostly submitted to compressive stresses, and should always be carefully evaluated for consolidation. For all other localizations, the risk of mechanical failure can be considered as 
much lower, especially if the cortical bone is intact, with in decreasing functional importance of the sacro-iliac, pubis, ischium and the iliac wing regions. The choice of optimal treatment should always be tailored to the clinical and radiological features of the tumor.

Conflict of interest statement: all authors declare that they do not have any conflict of interest

\section{Figures}

Fig.1: Representation of load transmission within the pelvis. (A) The loads are transmitted from the spine to the sacrum at the lumbosacral junction and then are transferred to the femur via the sacro-iliac joints and the hip joints. (B) Oblique sagittal CT-scan showing how the loads are transferred from the sacro-iliac joint to the acetabulum. (C) Same findings on an oblique coronal view.

Fig.2: Simplified schematic of the peak stresses in the pelvis during the 4 phases of walking. The acetabular area is constantly stressed, while the sacro-iliac areas and to a lesser degree the upper pubic rami are occasionally stressed. Figure adapted from [16]

Fig.3: Pelvic lesions with different clinical and radiological presentations and risks of pathologic fracture. (A) 3 osteolytic lesions in the posterior right iliac bone (asterisks), the left sacral wing (arrowheads) and the left iliac bone (arrow). The lesions are not located in weight bearing areas and there is no cortical destruction: the risk of mechanical failure is minimal. (B) Mixed pubic metastasis (arrows): there is no bone defect and the stresses are limited in that area, the risk of mechanical failure is very low. (C) Mixed metastasis with some kind of cortical destruction in a complete non-weight bearing location (the iliac wing): the risk of pathological 
fracture might be increased but prophylactic consolidation is not indicated (no risk of mechanical failure). Should a fracture occur, percutaneous treatment is a possibility (see below). (D) Osteolysis with cortical destruction in a critical mechanical area (the acetabulum): consolidation should be considered even if the patient is not symptomatic. (E) Multiple osteolytic lesions in a right pelvic bone: the lesions in the iliac wing (asterisks) do not require consolidation, while the acetabular metastasis (dotted line) interrupts the transmission of loads and should imperatively be consolidated. (F) Diffuse acetabular osteolysis with cortical and articular involvement: the risk of mechanical failure is high.

Fig.4: Sacro-iliac mechanical forces. Compression is the prevailing stress at the intermediate (A, coronal view) and anterior (B, axial view) part of the sacro-iliac joints. Tensile stresses predominate at the superior $(\mathrm{C}$, coronal view) and posterior $(\mathrm{D}$, axial view) part of the joints.

Fig.5: Consolidation of sacro-iliac lesions in patients with mechanical pain. (A) Axial CT-scan at the level of S1-S2 demonstrates an osteolytic lesion (asterisks) at the intermediate and anterior part of S1 and the right sacral ala. (B) CT at the L5-S1 level: there is no destruction of the superior and posterior part of the sacral ala. (C) The risk of fracture and failure to shear can be considered low: stand-alone cementoplasty was performed to provide resistance to compression. (D) Axial CT-scan at the S1-S2 level in another patient shows bilateral postradiation fractures of the sacrum (arrowheads). (E) The fracture is extending cranially with complete disruption of the posterior and superior part of the sacral ala, exposing to shear forces. (F) The fractures were stabilized with two trans sacro-iliac screws (one in S1 and one in S2) with additional cement injection to improve the anchorage of the screws. 
Fig.6: Acetabular loads. (A) The roof of the acetabulum (white area) is permanently stressed, while the anterior part (dark area) is typically only occasionally stressed and the posterior part (gray area) has a very limited role for load transmission. (B) Coronal and (C) axial CT-scan: the acetabular roof (dotted line) is key for the transfers of loads within the hip, with the lateral cortex (arrowheads) being the most important structure of the acetabulum.

Fig.7: Acetabular roof cementoplasty for consolidation. (A) Coronal CT-scan demonstrates a lytic metastasis in the acetabular roof (asterisk) without destruction of the articular surface and limited involvement of the lateral cortex. (B) Cementoplasty provides immediate consolidation. (C) Anteroposterior fluoroscopic projection and (D) axial CT-scan show a metastasis (black asterisk) with complete destruction of the lateral cortex (arrow) of the acetabulum resulting in high risk of mechanical failure. (E) Balloon kyphoplasty was used prior to cement injection to reduce the risk of leakage and the likehood of good filling $(F)(G)$ of osteolytic lesion and weightbearing portion of the supra-acetabular ilium.

Fig.8: Cementoplasty for extensive supra-acetabular osteolysis. (A) Axial CT- scan shows a large amount of osteolysis and destruction of the acetabular roof with minimal cortical integrity (asterisk). (B) sagittal oblique view shows a diffuse extension of the lesion. The roof of the acetabulum (dotted line) is at high risk of mechanical failure, while the posterior part (black asterisk) has very limited weight-bearing function. (C) and (D) The roof was filled as much as possible with cement while the posterior part was left untreated. Small asymptomatic anterior leakage in the soft tissue. (E) Axial CT-scan in another patient demonstrating a large supraacetabular osteolytic lesion (asterisk) with destruction of the lateral cortex (arrow). (F) Axial CT-scan more caudally shows that the medial wall of the acetabulum (asterisks) is also completely destroyed. (G) Large volume cementoplasty was performed as the patient was not 
a surgical candidate due to other comorbidities. $(\mathrm{H})$ One-year follow-up shows recalcification under systemic treatment. However, there is a complete destruction of the hip joint due with acetabular protrusion.

Fig.9: Combined screw fixation and cementoplasty to consolidate a complex acetabular pathological fracture. (A) Coronal oblique and (B) sagittal oblique CT-scan show a displaced pathological fracture of the acetabulum, in a patient not eligible for surgery. (C) AP fluoro projection and (D) sagittal oblique CT-scan: Cementoplasty was performed to reinforce the acetabular roof (asterisk) in combination with one retrograde transpubic screw (black arrow) and two anterior trans-iliac screws (white arrow) for fracture fixation. Partial mechanical improvement was noted after the intervention.

Fig.10: Screw fixation of a pathological iliac wing fracture. (A) Axial, (B) coronal and (C) VRT CT-scan show a large osteolytic metastasis with cortex destruction (white asterisk), responsible for a painful displaced fracture of the iliac wing with a mobile fragment (black asterisk). (D), (E), (F) Two screws were inserted perpendicular to the fracture line. Excellent immediate functional result.

\section{References}

1. Müller DA, Capanna R. The surgical treatment of pelvic bone metastases. Adv Orthop. 2015;2015:525363. doi: 10.1155/2015/525363. Epub 2015 Feb 24.

2. Deschamps F, de Baere T, Hakime A, Pearson E, et al. Percutaneous osteosynthesis in the pelvis in cancer patients. Eur Radiol. 2016 Jun;26(6):1631-9. doi: 10.1007/s00330015-3971-1 
3. Rich SE, Chow R, Raman S, Liang Zeng K, et al. Update of the systematic review of palliative radiation therapy fractionation for bone metastases. Radiother Oncol. 2018 Mar;126(3):547-557. doi:10.1016/j.radonc.2018.01.003.

4. Erler D, Brotherston D, Sahgal A, Cheung P, et al. Local control and fracture risk following stereotactic body radiation therapy for non-spine bone metastases. Radiother Oncol. 2018 May;127(2):304-309. doi: 10.1016/j.radonc.2018.03.030.

5. Wunder JS, Ferguson PC, Griffin AM, Pressman A, Bell RS. Acetabular metastases: planning for reconstruction and review of results. Clin Orthop Relat Res. 2003 Oct;(415 Suppl):S187-97.

6. Spinelli MS, Ziranu A, Piccioli A, Maccauro G. Surgical treatment of acetabular metastasis. Eur Rev Med Pharmacol Sci. 2016 Jul;20(14):3005-10.

7. Brown TS, Salib CG, Rose PS, Sim FH, et al. Reconstruction of the hip after resection of periacetabular oncological lesions: a systematic review. Bone Joint J. 2018 Jan;100B(1 Supple A):22-30. doi:10.1302/0301-620X.100B1.BJJ-2017-0548.R1.

8. Mastier C, Gjorgjievska A, Thivolet A, Bouhamama A, et al. Musculoskeletal Metastases Management: The Interventional Radiologist's Toolbox. Semin Intervent Radiol. 2018 Oct;35(4):281-289. doi: 10.1055/s-0038-1673420.

9. Roux C, Tselikas L, Yevich S, Sandes Solha R, et al. Fluoroscopy and Cone-Beam CTguided Fixation by Internal Cemented Screw for Pathologic Pelvic Fractures. Radiology. 2019 Feb;290(2):418-425. doi: 10.1148/radiol.2018181105.

10. Kurup AN, Morris JM, Schmit GD, Atwell TD, et al. Balloon-assisted osteoplasty of periacetabular tumors following percutaneous cryoablation. J Vasc Interv Radiol. 2015 Apr;26(4):588-94. doi:10.1016/j.jvir.2014.11.023. 
11. Wobser AM, Wobser RW. Anatomy, Abdomen and Pelvis, Bones (Ilium, Ischium, and Pubis). 2018 Dec 6. StatPearls [Internet]. Treasure Island (FL): StatPearls Publishing; 2019 Jan-.

12. Dalstra M, Huiskes R. Load transfer across the pelvic bone. J Biomech. 1995 Jun;28(6):715-24.

13. Polkowski GG, Clohisy JC. Hip biomechanics. Sports Med Arthrosc Rev. 2010 Jun;18(2):56-62. doi: 10.1097/JSA.0b013e3181dc5774.

14. Dalstra M, Huiskes R, Odgaard A, van Erning L. Mechanical and textural properties of pelvic trabecular bone. J Biomech. 1993 Apr-May;26(4-5):523-35.

15. Hammer N, Steinke H, Lingslebe U, Bechmann I, et al. Ligamentous influence in pelvic load distribution. Spine J. 2013 Oct;13(10):1321-30. doi: 10.1016/j.spinee.2013.03.050.

16. Volinski B, Kalra A, Yang K. Evaluation of full pelvic ring stresses using a bilateral static gait-phase finite element modeling method. J Mech Behav Biomed Mater. 2018 Feb;78:175-187. doi: 10.1016/j.jmbbm.2017.11.006.

17. Dalstra M, Huiskes R, Odgaard A, van Erning L. Mechanical and textural properties of pelvic trabecular bone. J Biomech. 1993 Apr-May;26(4-5):523-35.

18. Jin T, Liu $\mathrm{W}, \mathrm{Xu} \mathrm{H}, \mathrm{Li} \mathrm{Y}$, et al. How does iliosacral bone tumor resection without reconstruction affect the ipsilateral hip joint? BMC Musculoskelet Disord. 2018 Apr 4;19(1):102. doi: 10.1186/s12891-018-2023-9

19. Wang B, Zou C, Hu X, Tu J, et al. Reconstruction with a novel combined hemipelvic endoprosthesis after resection of periacetabular tumors involving the sacroiliac joint: a report of 25 consecutive cases. BMC Cancer. 2019 Aug 30;19(1):861. doi: 10.1186/s12885-019-6049-7. 
20. Arjmand N, Shirazi-Adl A. Role of intra-abdominal pressure in the unloading and stabilization of the human spine during static lifting tasks. Eur Spine J.2006 Aug;15(8):1265-75.

21. Vleeming A, Schuenke MD, Masi AT, Carreiro JE, et al. The sacroiliac joint: an overview of its anatomy, function and potential clinical implications. J Anat. 2012 Dec;221(6):537-67. doi:10.1111/j.1469-7580.2012.01564.x.

22. Munro JT, Fernandez JW, Millar JS, Walker CG, et al. Altered load transfer in the pelvis in the presence of periprosthetic osteolysis. J Biomech Eng. 2014 Nov;136(11). doi: $10.1115 / 1.4028522$.

23. Garnon J, Meylheuc L, Cazzato RL, Dalili D, et al. Percutaneous extra-spinal cementoplasty in patients with cancer: A systematic review of procedural details and clinical outcomes. Diagn Interv Imaging. 2019 Aug 16. pii: S2211-5684(19)30171-8. doi: 10.1016/j.diii.2019.07.005.

24. Lewis G. Viscoelastic properties of injectable bone cements for orthopaedic applications: state-of-the-art review. J Biomed Mater Res B Appl Biomater. 2011 Jul;98(1):171-91. doi: 10.1002/jbm.b.31835. Epub 2011 Apr 18.

25. Delpla A, Tselikas L, De Baere T, Laurent S, et al. Preventive Vertebroplasty for LongTerm Consolidation of Vertebral Metastases. Cardiovasc Intervent Radiol. 2019 Aug 23. doi: 10.1007/s00270-019-02314-6.

26. Li Z, Butala NB, Etheridge BS, Siegel HJ, et al. A biomechanical study of periacetabular defects and cement filling. J Biomech Eng. 2007 Apr;129(2):129-36.

27. Cazzato RL, Garnon J, Tsoumakidou G, Koch G, et al. Percutaneous image-guided screws meditated osteosynthesis of impeding and pathological/insufficiency fractures of the femoral neck in non-surgical cancer patients. Eur J Radiol. 2017 May;90:1-5. doi: 10.1016/j.ejrad.2017.02.022. 
28. Deschamps F, Farouil G, Hakime A, Teriitehau C, et al. Percutaneous stabilization of impending pathological fracture of the proximal femur. Cardiovasc Intervent Radiol. 2012 Dec;35(6):1428-32. doi:10.1007/s00270-011-0330-8.

29. Lea WB, Neilson JC, King DM, Tutton SM. Minimally Invasive Stabilization Using Screws and Cement for Pelvic Metastases: Technical Considerations for the Pelvic "Screw and Glue" Technique. Semin Intervent Radiol. 2019 Aug;36(3):229-240. doi: $10.1055 / \mathrm{s}-0039-1693982$.

30. Deschamps F, Yevich S, Gravel G, Roux C, et al. Percutaneous Fixation by Internal Cemented Screw for the Treatment of Unstable Osseous Disease in Cancer Patients. Semin Intervent Radiol. 2018 Oct;35(4):238-247. doi: 10.1055/s-0038-1673359.

31. Sattar MH, Guthrie ST. Anatomy, Back, Sacral Vertebrae. 2019 Nov 28. StatPearls [Internet]. Treasure Island (FL): StatPearls Publishing; 2020 Jan-.Available from http://www.ncbi.nlm.nih.gov/books/NBK551653/

32. Betts A. Vertebroplasty of the first sacral vertebra. Pain Physician. 2009 MayJun;12(3):651-7.

33. Rohlmann A, Boustani HN, Bergmann G, Zander T. A probabilistic finite element analysis of the stresses in the augmented vertebral body after vertebroplasty. Eur Spine J. 2010 Sep;19(9):1585-95. doi: 10.1007/s00586-010-1386-х.

34. Kiapour A, Joukar A, Elgafy H, Erbulut DU, et al. Biomechanics of the Sacroiliac Joint: Anatomy, Function, Biomechanics, Sexual Dimorphism, and Causes of Pain. Int J Spine Surg. 2020 Feb 10;14(Suppl 1):3-13. doi:10.14444/6077.

35. Snijders CJ, Vleeming A, Stoeckart R. Transfer of lumbosacral load to iliac bones and legs Part 1: Biomechanics of self-bracing of the sacroiliac joints and its significance for treatment and exercise. Clin Biomech (Bristol, Avon). 1993 Nov;8(6):285-94. doi: 10.1016/0268-0033(93)90002-Y. 
36. Frey ME, Warner C, Thomas SM, Johar K, et al. Sacroplasty: A Ten-Year Analysis of Prospective Patients Treated with Percutaneous Sacroplasty: Literature Review and Technical Considerations. Pain Physician. 2017 Nov;20(7):E1063-E1072.

37. Moussazadeh N, Laufer I, Werner T, Krol G, et al. Sacroplasty for cancer-associated insufficiency fractures. Neurosurgery. 2015 Apr;76(4):446-50; discussion 450. doi: 10.1227/NEU.0000000000000658.

38. Chandra V, Wajswol E, Shukla P, Contractor S, Kumar A. Safety and Efficacy of Sacroplasty for Sacral Fractures: A Systematic Review and Meta-Analysis. J Vasc Interv Radiol. 2019 Nov;30(11):1845-1854. doi: 10.1016/j.jvir.2019.06.013.

39. Walker JB, Mitchell SM, Karr SD, Lowe JA, Jones CB. Percutaneous TransiliacTranssacral Screw Fixation of Sacral Fragility Fractures Improves Pain, Ambulation, and Rate of Disposition to Home. J Orthop Trauma. 2018 Sep;32(9):452-456. doi: 10.1097/BOT.0000000000001243.

40. Papathanasopoulos A, Tzioupis C, Giannoudis VP, Roberts C, Giannoudis PV. Biomechanical aspects of pelvic ring reconstruction techniques: Evidence today. Injury. 2010 Dec;41(12):1220-7. doi: 10.1016/j.injury.2010.10.001.

41. Lee CH, Hsu CC, Huang PY. Biomechanical study of different fixation techniques for the treatment of sacroiliac joint injuries using finite element analyses and biomechanical tests. Comput Biol Med. 2017 Aug 1;87:250-257. doi:10.1016/j.compbiomed.2017.06.007.

42. Hu P, Wu T, Wang HZ, Qi XZ, et al. Influence of Different Boundary Conditions in Finite Element Analysis on Pelvic Biomechanical Load Transmission. Orthop Surg. 2017 Feb;9(1):115-122. doi: 10.1111/os.12315. Epub 2017 Mar 16. 
43. Ghosh R, Pal B, Ghosh D, Gupta S. Finite element analysis of a hemi-pelvis: the effect of inclusion of cartilage layer on acetabular stresses and strain. Comput Methods Biomech Biomed Engin. 2015;18(7):697-710. doi: 10.1080/10255842.2013.843674.

44. Issack PS, Kotwal SY, Lane JM. Management of metastatic bone disease of the acetabulum. J Am Acad Orthop Surg. 2013 Nov;21(11):685-95. doi: 10.5435/JAAOS21-11-685.

45. Charles T, Ameye L, Gebhart M. Surgical treatment for periacetabular metastatic lesions. Eur J Surg Oncol. 2017 Sep;43(9):1727-1732. doi: 10.1016/j.ejso.2017.03.018.

46. Kurup AN, Schmit GD, Atwell TD, Sviggum EB, et al. Palliative Percutaneous Cryoablation and Cementoplasty of Acetabular Metastases: Factors Affecting Pain Control and Fracture Risk. Cardiovasc Intervent Radiol. 2018 Nov;41(11):1735-1742. doi: 10.1007/s00270-018-1998-9.

47. Friedman MV, Hillen TJ, Wessell DE, Hildebolt CF, Jennings JW. Hip chondrolysis and femoral head osteonecrosis: a complication of periacetabular cryoablation. J Vasc Interv Radiol. 2014 Oct;25(10):1580-8. doi:10.1016/j.jvir.2014.06.016.

48. Vielgut I, Sadoghi P, Gregori M, Kovar FM, et al. The modified Harrington procedure for metastatic peri-acetabular bone destruction. Int Orthop. 2013 Oct;37(10):1981-5. doi: 10.1007/s00264-013-1940-3. Epub 2013 Jun 12.

49. Coupal TM, Pennycooke K, Mallinson PI, Ouellette HA, et al. The Hopeless Case? Palliative Cryoablation and Cementoplasty Procedures for Palliation of Large Pelvic Bone Metastases. Pain Physician. 2017 Nov;20(7):E1053-E1061.

50. Fensky F, Weiser L, Sellenschloh K, Vollmer M, et al. Biomechanical analysis of anterior pelvic ring fractures with intact peripelvic soft tissues: a cadaveric study. Eur J Trauma Emerg Surg. 2019 Aug 28. doi: 10.1007/s00068-019-01213-2. 
51. Rommens PM, Graafen M, Arand C, Mehling I, et al. Minimal-invasive stabilization of anterior pelvic ring fractures with retrograde transpubic screws. Injury. 2020 Feb;51(2):340-346. doi: 10.1016/j.injury.2019.12.018.

52. Dimitriou R, Mataliotakis GI, Angoules AG, Kanakaris NK, Giannoudis PV. Complications following autologous bone graft harvesting from the iliac crest and using the RIA: a systematic review. Injury. 2011 Sep;42 Suppl 2:S3-15. doi:10.1016/j.injury.2011.06.015.

53. Schmitz P, Cornelius Neumann C, Neumann C, Nerlich M, Dendorfer S. Biomechanical analysis of iliac crest loading following cortico-cancellous bone harvesting. J Orthop Surg Res. 2018 May 9;13(1):108. doi:10.1186/s13018-018-0822-1.

54. Kim WY, Lee SW, Kim KW, Kwon SY, Choi YH. Minimally invasive surgical treatment using 'iliac pillar' screw for isolated iliac wing fractures in geriatric patients: a new challenge. Eur J Trauma Emerg Surg. 2019 Apr;45(2):213-219. doi: $10.1007 / \mathrm{s} 00068-018-1046-0$.

55. Tsoumakidou G, Borensztein M, Zini C, Garnon J, Gangi A. Postablation insufficiency fracture of the iliac crest: management by percutaneous screw fixation. Cardiovasc Intervent Radiol. 2014 Aug;37(4):1126-8. doi: 10.1007/s00270-013-0781-1. 